\title{
DNA ploidy in curettage specimens identifies high-risk patients and lymph node metastasis in endometrial cancer
}

T S Njølstad ${ }^{1,2}$, J Trovik 1,2, T S Hveem 3,4,5, M L Kjæreng ${ }^{3,4}$, W Kildal ${ }^{3,4}$, M Pradhan 3,4, J Marcickiewicz ${ }^{6,7}$, S Tingulstad ${ }^{8}$, A C Staff ${ }^{9}, \mathrm{H}$ K Haugland ${ }^{10}$, R Eraker ${ }^{11,12}$, K Oddenes $^{13}$, J A Rokne ${ }^{14}$, J Tjugum ${ }^{15}$, M S Lode L $^{16}$, ENITEC Network/MoMaTEC Study Group ${ }^{19}$, F Amant ${ }^{17}$, H M Werner ${ }^{1,2}$, H B Salvesen ${ }^{1,2}$ and H E Danielsen ${ }^{\star}, 3,4,5,18$

${ }^{1}$ Department of Clinical Science, University of Bergen, Haukeland University Hospital, 5021 Bergen, Norway; ${ }^{2}$ Department of Obstetrics and Gynecology, Haukeland University Hospital, 5021 Bergen, Norway; ${ }^{3}$ Institute for Cancer Genetics and Informatics, Oslo University Hospital, 0424 Oslo, Norway; ${ }^{4}$ Centre for Cancer Biomedicine, University of Oslo, 0424 Oslo, Norway; ${ }^{5}$ Department of Informatics, University of Oslo, 0310 Oslo, Norway; ${ }^{6}$ Department of Gynecology, Sahlgrenska Academy, University of Gothenburg, S-413 45 Gothenburg, Sweden; ${ }^{7}$ Department of Obstetrics and Gynecology, Halland's Hospital Varberg, 43237 Varberg, Sweden; ${ }^{8}$ Department of Gynecology, St Olav's Hospital, 7006 Trondheim, Norway; ${ }^{9}$ Department of Gynecology, Oslo University Hospital, Ulleval, 0424 Oslo, Norway; ${ }^{10}$ Department of Pathology, The Gade Institute, Haukeland University Hospital, 5021 Bergen, Norway; ${ }^{11}$ Department of Obstetrics and Gynecology, Akershus University Hospital, 1478 Lørenskog, Norway; ${ }^{12}$ University of Oslo, Oslo, Norway; ${ }^{13}$ Department of Obstetrics and Gynecology, Haugesund Hospital, 5528 Haugesund, Norway; ${ }^{14}$ Department of Obstetrics and Gynecology, Hospital of Vestfold, 3116 Tønsberg, Norway; ${ }^{15}$ Department of Obstetrics and Gynecology, Førde Hospital, 6800 Førde, Norway; ${ }^{16}$ Department of Obstetrics and Gynecology, Ålesund Hospital, 6017 Ålesund, Norway; ${ }^{17}$ Department of Gynecologic Oncology, UZGasthuisberg, KULeuven, 3000 Leuven, Belgium and ${ }^{18}$ Nuffield Division of Clinical Laboratory Sciences, University of Oxford, Oxford, UK

Background: Preoperative risk stratification is essential in tailoring endometrial cancer treatment, and biomarkers predicting lymph node metastasis and aggressive disease are aspired in clinical practice. DNA ploidy assessment in hysterectomy specimens is a well-established prognostic marker. DNA ploidy assessment in preoperative curettage specimens is less studied, and in particular in relation to the occurrence of lymph node metastasis.

Methods: Curettage image cytometry DNA ploidy in relation to established clinicopathological variables and outcome was investigated in 785 endometrial carcinoma patients prospectively included in the MoMaTEC multicentre trial.

Results: Diploid curettage status was found in $72.0 \%$, whereas $28.0 \%$ were non-diploid. Non-diploid status significantly correlated with traditional aggressive postoperative clinicopathological features, and was an independent predictor of lymph node metastasis among FIGO stage I-III patients in multivariate analysis (OR 1.94, $P=0.033)$. Non-diploid status was related to shorter disease-specific survival (5-year DSS of $74.4 \%$ vs $88.8 \%$ for diploid curettage, $P<0.001$ ). When stratifying by FIGO stage and lymph node status, the prognostic effect remained. However, in multivariate regression analysis, preoperative histological risk classification was a stronger predictor of DSS than DNA ploidy.

Conclusions: Non-diploid curettage is significantly associated with aggressive clinicopathological phenotype, lymph node metastasis, and poor survival in endometrial cancer. The prognostic effect was also observed among subgroups with (presumably) less aggressive traits, such as low FIGO stage and negative lymph node status. Our results indicate curettage DNA ploidy as a possible supplement to existing parameters used to tailor surgical treatment.

*Correspondence: Professor HE Danielsen; E-mail: hdaniels@labmed.uio.no

${ }^{19}$ See Appendix.

Received 5 December 2014; revised 24 February 2015; accepted 8 March 2015; published online 21 April 2015

(c) 2015 Cancer Research UK. All rights reserved 0007-0920/15 
Endometrial carcinoma is the most common gynaecological malignancy in the western world with a reported lifetime risk of $2-3 \%$ (Jemal et al, 2010). In Norway, it constitutes 6\% of all female cancers. A steady increase in incidence over the past decades has been observed (Cancer Registry of Norway, 2012), attributed to an ageing population and increasing incidence of obesity (Sorosky, 2012). In the United States, estimates by The American Cancer Society predict 52630 new cases and 8590 endometrial cancer deaths in 2014 (American Cancer Society, 2014; Siegel et al, 2014).

Abnormal uterine bleeding is the cardinal symptom of endometrial cancer. Essential preoperative diagnostic work-up includes histological verification by endometrial biopsy or curettage, in combination with a selection of imaging techniques to predict metastatic disease. Surgery remains the cornerstone in primary treatment; with curative intent it includes total hysterectomy and bilateral salpingo-oophorectomy, with or without retroperitoneal lymph node dissection and omentectomy, dependent on risk assessment based on tumour characteristics as well as patient operability (Amant et al, 2005).

Prognostic factors in endometrial cancer have been thoroughly investigated, the most important being age, the surgical FIGO stage (International Federation of Gynaecology and Obstetrics), myometrial invasion, histological subtype and grade, and lymphovascular invasion (Creutzberg et al, 2000; Prat, 2004; Amant et al, 2005), serving as important determinants of tailoring postoperative treatment. Fortunately, the majority of endometrial cancer patients are diagnosed at a relatively early stage with disease presumably confined to the uterus. This is reflected by a generally favourable prognosis, with a 5-year relative survival of $84.4 \%$ reported for the period 2008-2010 in Norway (Cancer Registry of Norway, 2012), and 5-year disease-specific survival (DSS) reported to be $86.9 \%$ (Trovik et al, 2012). However, endometrial carcinoma is a heterogeneous disease with great variations in aggressiveness, exemplified by a recurrence rate after primary surgery among patients with presumed localised disease of 15-20\% (Abeler and Kjorstad, 1991). Consequently, much effort has been made to individualise treatment by (preferably preoperatively) more accurately identifying patients with higher risk for poor outcome requiring more aggressive treatment, as well as identifying low-risk patients to avoid overtreatment (Salvesen et al, 2012). As reviewed by Salvesen et al (2012), today's preoperative risk assessment based on endometrial histology and imaging could be further augmented by preoperatively accessible biomarkers, such as curettage specimen hormone receptor status (Trovik et al, 2013), stathmin expression (Trovik et al, 2011), and DNA ploidy (FredstorpLidebring et al, 2001; Mariani et al, 2000; Steinbakk et al, 2011; Pradhan et al, 2012) for tailored surgical treatment.

Assessment of DNA ploidy status has been shown to be of prognostic value in several epithelial cancers (Merkel and McGuire, 1990). For endometrial cancer patients, DNA ploidy postoperatively acquired from hysterectomy specimens has repeatedly been reported to be of prognostic importance, with aneuploidy or nondiploidy as a marker for aggressive disease (Pfisterer et al, 1995; Zaino et al, 1998; Larson et al, 1999; Salvesen and Akslen, 2002; Lundgren et al, 2004; Susini et al, 2007; Wik et al, 2009; Pradhan et al, 2012). However, only few studies have assessed the value of DNA ploidy in preoperatively available curettage specimens, both in terms of prognosis (Mariani et al, 2000; Fredstorp-Lidebring et al, 2001; Steinbakk et al, 2011; Pradhan et al, 2012) and in relation to lymph node status (Mariani et al, 2005). As results from multivariate analyses are conflicting (Terada, 2012; Mauland et al, 2014), the clinical value of DNA ploidy assessment needs further validation.

On this background, we have investigated DNA ploidy in curettage specimens in relation to a panel of clinicopathological variables, lymph node status and outcome for women diagnosed and treated for endometrial cancer in the prospective, international multicentre trial MoMaTEC1 (Molecular Markers in Treatment of Endometrial Cancer).

MATERIALS AND METHODS

Study cohort. With Regional Ethics Committee approval, a total of 1046 consenting patients in the MoMaTEC1 trial (Clinical Trial identifier NCT00598845) were included in this study. The study has acquired approval of The Norwegian Data Inspectorate (961478-2), Norwegian Social Sciences Data services (15501), and Regional Committees for Medical and Health Research Ethics (REKIII no. 052.01). A total of 402 endometrial cancer patients were prospectively included when treated at the Department of Gynaecology and Obstetrics, Haukeland University Hospital, Bergen, Norway, from May 2001 to March 2011. Nine other centres contributed with 644 patients treated for endometrial carcinoma prospectively included at their institutions. Sampling was performed by pipelle or dilatation and curettage as per routine for each contributing centre. Formalin-fixed, paraffin-embedded tumour tissue from curettage specimens was collected from all participating institutions.

Clinicopathological data, including age at diagnosis, FIGO stage according to 2009 criteria, histology (type and grade) from hysterectomy specimens, and treatment modalities were recorded. Preoperative curettage histology reports were routinely categorised as either high risk (standardly comprising histological type reported as non-endometrioid, or histological grade 3 endometrioid carcinoma) or low risk (standardly comprising other histological diagnoses including grade 1 or 2 endometrioid carcinoma, hyperplasia, and benign endometrium). All precision samples selected for DNA ploidy analysis as later described were investigated for presence of malignant tissue by one of the coauthors (MP), irrespective of the preoperative routine histological diagnosis. Complying with the aim to evaluate DNA ploidy impact in a patient group treated routinely in a prospective multicentre setting, routine histological reports for grading and subtyping were applied from the participating centres. Follow-up data with records of recurrence and survival were collected from patient records and correspondence with physicians responsible for outpatient controls.

DNA ploidy analyses. Haematoxylin and eosin-stained slides from individual curettage specimen were evaluated to identify tissue areas with highest tumour grade. From the selected area, one or two $1 \mathrm{~mm}$ cylinders were punched out using a custom-made precision instrument (Beecher Instruments, Silver Spring, MD, USA) depending on the curettage tissue depth. Prepared monolayers were stained with Feulgen-Schiff as previously described by Pradhan et al (2006). The nuclear DNA content was measured using the Ploidy Work Station (PWS) Grabber version 1.4.12 (Room4 Ltd, Crowborough, East Sussex, UK) and a Zeiss Axioplan microscope equipped with a 546-nm green filter and a black-andwhite high-resolution digital camera (Axiocam MRM, Zeiss, Jena, Germany). DNA ploidy histograms were created based on Integrated Optical Density of the nuclei using PWS Classifier (vs 3.06.03, Room4 Ltd). DNA ploidy histograms were classified as diploid (one $\mathrm{G}_{0} / \mathrm{G}_{1}$ peak and $\mathrm{G}_{2}<10 \%$ ), aneuploid (DNA Index (DI) $1.06-1.89,2.11-3.79$ or $>4.2$ ), tetraploid (DI 1.90-2.10), or polyploid (DI 3.8-4.2). A detailed description of the procedure, DNA content measurement, and histogram classification criteria is given elsewhere (Pradhan et al, 2006). Samples with $<200$ nuclei, and samples with poor technical quality were not classified.

Statistical analysis. For statistical analysis, SPSS, Statistical Package for the Social Sciences, version 20.0 (IBM, Chicago, IL, USA) was applied. Associations between categorical variables were explored using Pearson's $\chi^{2}$ and odds ratios (ORs) for lymph node 
metastasis by binary logistic regression. For evaluation of prognostic impact, DSS was defined as time from surgery until death from endometrial carcinoma, as documented from each patient's responsible clinician. Univariate survival analyses were conducted by the Kaplan-Meier method with log-rank test for statistical significance, and multivariate survival analyses by Cox regression. All tests were two sided, and results were considered statistically significant with a probability of $<0.05$.

\section{RESULTS}

Of 1046 curettage samples, 236 samples were discarded because of lack of sufficient tissue material, being not measurable, or unclassifiable owing to poor technical quality. Additional 25 patients had incomplete clinicopathological data upon study completion, leaving 785 patients for the final analysis. Follow-up information was available for 733 patients, with a mean follow-up among survivors of 38.4 months (range 0-96 months).

Of these 785 patients, $97.2 \%(n=763)$ were treated with primary hysterectomy and bilateral salpingo-oopherectomy, including lymph node staging in $74.4 \%$ of the cases $(n=584)$, $2.4 \%(n=19)$ were treated by curettage only, and $0.4 \%(n=3)$ by cytoreductive surgery. Lymph node sampling was predominantly pelvic, but included para-aortic dissection for a minority of patients $(n=27)$. Performed lymph node dissection was significantly associated with younger age $(82.6 \%$ of patients under 66 years $v s 66.5 \%$ of patients over 66 years, $P<0.001$ ), but showed no significant association to preoperative histological risk classification or curettage DNA ploidy (Supplementary Table 1). In addition, $33.6 \%$ of the patients $(n=264)$ received adjuvant therapy, consisting of various combinations of radiation $(n=115)$, hormonal treatment $(n=13)$, and/or chemotherapy $(n=171)$. The study complied with standardly applied treatment protocols for adjuvant therapy at the including centre, individually adjusted for each patient by the responsible physicians. Hormonal treatment was given to 13 patients because of high age (median 86 years) and/or inoperability. None of the patients had a prior history of endometrial cancer, and none received neoadjuvant radiation or chemotherapy.

Evaluating DNA ploidy from curettage samples, $72.0 \%$ $(n=565)$ were classified as diploid, $20.6 \%(n=162)$ as aneuploid, $6.6 \%(n=52)$ as tetraploid, and $0.8 \%(n=6)$ as polyploid. Dichotomising patients according to diploid and non-diploid curettage specimens, aneuploid, tetraploid, and polyploidy tumours were merged, constituting $28.0 \%$ of total patients included. Details for clinicopathological and demographic characteristics of the study cohort are presented in Table 1. Distribution of preoperative and postoperatively available clinicopathological findings in relation to DNA ploidy status are presented in Table 2. Non-diploid curettage specimen was significantly associated with high FIGO stage, non-endometrioid histological subtype in hysterectomy specimen, high histological grade in hysterectomy specimen, and high-risk curettage classification (all $P<0.001$ ). Associations between lymph node status, clinicopathological variables, and DNA ploidy are presented in Table 3. Interestingly, patients with nondiploid curettage had significantly higher occurrence of lymph node metastasis compared with patients with diploid curettage $(20.6 \%$ vs $9.1 \%$ respectively, $P<0.001)$. High-risk curettage classification was also associated with lymph node metastasis compared with low-risk curettage $(24.8 \%$ vs $8.6 \%$ respectively, $P<0.001)$, in concordance with previously established research findings and clinical practice for identifying high-risk patients for more extensive lymphadenectomy.

Biopsy reports were routinely categorised as either low or high risk for clinical risk stratification, as previously described (Trovik et al, 2011). Among patients with FIGO stages I-III, non-diploid
Table 1. Clinicopathological and demographic characteristics for 785 endometrial cancer MoMaTEC ${ }^{a}$ trial patients

\begin{tabular}{|l|c|c|}
\hline Characteristics & Mean & s.d., min-max \\
\hline Age (years) & 66.4 & $11.1,28-98$ \\
\hline Parity & 2.2 & $1.4,0-8$ \\
\hline Characteristics & Number of cases & Percentage \\
\hline Information available preoperatively & \multicolumn{2}{|l}{} \\
\hline Curettage DNA ploidy & 565 & 72.0 \\
Diploid & 162 & 20.6 \\
Aneuploid & 52 & 6.6 \\
Tetraploid & 6 & 0.8 \\
Polyploid & &
\end{tabular}

\section{Information available postoperatively}

Histological subtype

Endometrioid carcinomad

Serous carcinoma

Clear cell carcinoma

Carcinosarcoma

Undifferentiated carcinoma/other

Histological differentiation ${ }^{\mathrm{e}}$

Grade 1

Grade 2

Grade 3

Myometrial invasion $^{f}$

$<50 \%$

$\geq 50 \%$

FIGO stage (2009 revision)

I

II

III

Lymph node metastasis $^{\mathrm{g}}$

No

Yes

6

Abbreviation: FIGO = International Federation of Gynaecology and Obstetrics.

${ }^{a}$ Molecular Markers in Treatment of Endometrial Cancer.

${ }^{b}$ Parity information missing for 10 patients.

${ }^{c_{H}}$ Histological subtype missing for two patients.

$\mathbf{d}_{\text {Including cases with squamous differentiation. }}$

e Histological differentiation missing for five patients.

fData for myometrial infiltration not available for 77 patients.

$\mathbf{g}_{\text {Lymph node status evaluated in } 584 \text { patients. }}$

curettage specimens significantly predicted the occurrence of lymph node metastasis in both univariate analysis (OR 2.73, 95\% CI: $1.60-4.66, P<0.001)$ and multivariate analysis when adjusted for reported preoperative curettage risk stratification (OR 1.94, 95\% CI: $1.06-3.55, P=0.033$ ) (Table 4). However, the longestablished high-risk histology classification had a similar association to positive lymph node status (OR 2.23, 95\% CI: 1.20-4.13, $P=0.011$ ) (Table 4).

In univariate survival analysis, results from surgical staging incorporating final histpoathological diagnosis, FIGO stage, depth of myometrial infiltration, and lymph node status were, as expected, statistically significant predictors of DSS (Table 5). Among preoperatively available information, age, DNA ploidy, and histological risk classification showed significant associations. Patients with non-diploid curettage specimens had significantly shorter 5-year DSS compared with patients with diploid curettage (74.4\% compared with $89.6 \% 5$-year DSS, $P<0.001$; Figure 1A). A similar pattern was found when analysing FIGO stage I and II patients in isolation, with a 5-year DSS of $95.4 \%$ for diploid curettage vs $86.3 \%$ for non-diploid curettage $(P<0.001$; Supplementary Table 2 and Figure 1B). Noticeably, when viewing the subgroup of women with high FIGO stage (III and IV), no significant difference in ploidy-dependent survival was observed, with 5-year DSS for diploid and non-diploid curettage of $41.4 \%$ and $38.1 \%$ respectively $(P=0.267)$. 
Table 2. Clinicopathological variables related to DNA ploidy in curettage specimens from 785 patients with endometrial cancer, classified as either diploid or non-diploid (aneuploid, tetraploid, and polyploid)

\begin{tabular}{|c|c|c|c|c|c|c|}
\hline \multirow{2}{*}{$\begin{array}{l}\text { Variables } \\
\text { Age at primary treatment }\end{array}$} & \multirow{2}{*}{$\begin{array}{l}\text { Category } \\
<66 \text { Years }\end{array}$} & \multicolumn{2}{|c|}{ Diploid } & \multicolumn{2}{|c|}{ Non-diploid } & \multirow[t]{2}{*}{$P$-value } \\
\hline & & 309 & $80.3 \%$ & 76 & $19.7 \%$ & \\
\hline & $\geq 66$ Years & 256 & $64.0 \%$ & 144 & $36.0 \%$ & $<0.001$ \\
\hline \multicolumn{7}{|l|}{ Information available preoperatively } \\
\hline Curettage histology classification $^{\mathbf{a}}$ & $\begin{array}{l}\text { Low risk } \\
\text { High risk }\end{array}$ & $\begin{array}{r}499 \\
63\end{array}$ & $\begin{array}{l}83.2 \% \\
35.0 \%\end{array}$ & $\begin{array}{l}101 \\
117\end{array}$ & $\begin{array}{l}16.8 \% \\
65.0 \%\end{array}$ & $<0.001$ \\
\hline \multicolumn{7}{|l|}{ Information available postoperatively } \\
\hline 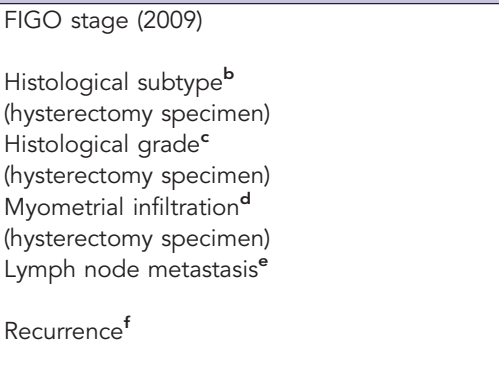 & $\begin{array}{c}\text { I/II } \\
\text { III/IV } \\
\text { Endometrioid } \\
\text { Non-endometrioid } \\
\text { Grades } 1 \text { and } 2 \\
\text { Grade 3 } \\
<50 \% \\
\geq 50 \% \\
\text { No } \\
\text { Yes } \\
\text { No } \\
\text { Yes }\end{array}$ & $\begin{array}{r}503 \\
62 \\
530 \\
35 \\
462 \\
101 \\
349 \\
173 \\
381 \\
38 \\
443 \\
58\end{array}$ & $\begin{array}{l}75.5 \% \\
52.1 \% \\
80.8 \% \\
27.6 \% \\
83.5 \% \\
44.5 \% \\
77.0 \% \\
67.8 \% \\
74.4 \% \\
52.8 \% \\
76.5 \% \\
57.4 \%\end{array}$ & $\begin{array}{r}163 \\
57 \\
126 \\
92 \\
91 \\
126 \\
104 \\
82 \\
131 \\
34 \\
136 \\
43\end{array}$ & $\begin{array}{l}24.5 \% \\
47.9 \% \\
19.2 \% \\
72.4 \% \\
16.5 \% \\
55.5 \% \\
23.0 \% \\
32.2 \% \\
25.6 \% \\
47.2 \% \\
23.5 \% \\
42.6 \%\end{array}$ & $\begin{array}{l}<0.001 \\
<0.001 \\
<0.001 \\
0.008 \\
<0.001 \\
<0.001\end{array}$ \\
\hline \multicolumn{7}{|c|}{ 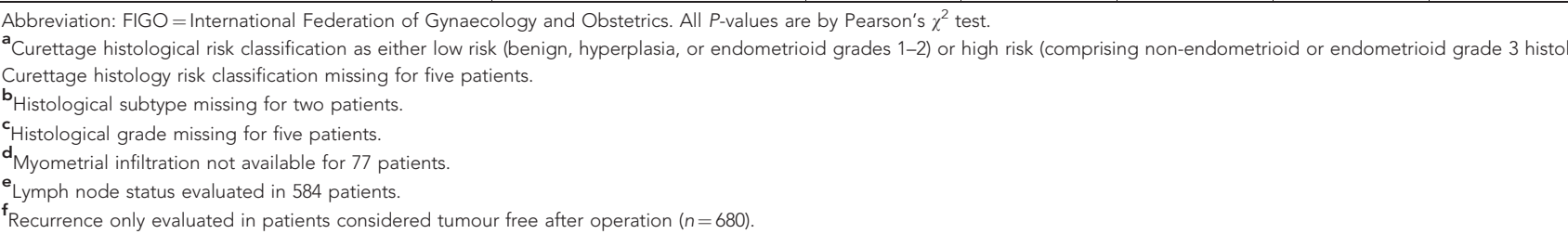 } \\
\hline
\end{tabular}

Table 3. Lymph node status in 584 endometrial cancer patients subjected to lymphadenectomy in relation to clinicopathological variables and expression of biomarkers

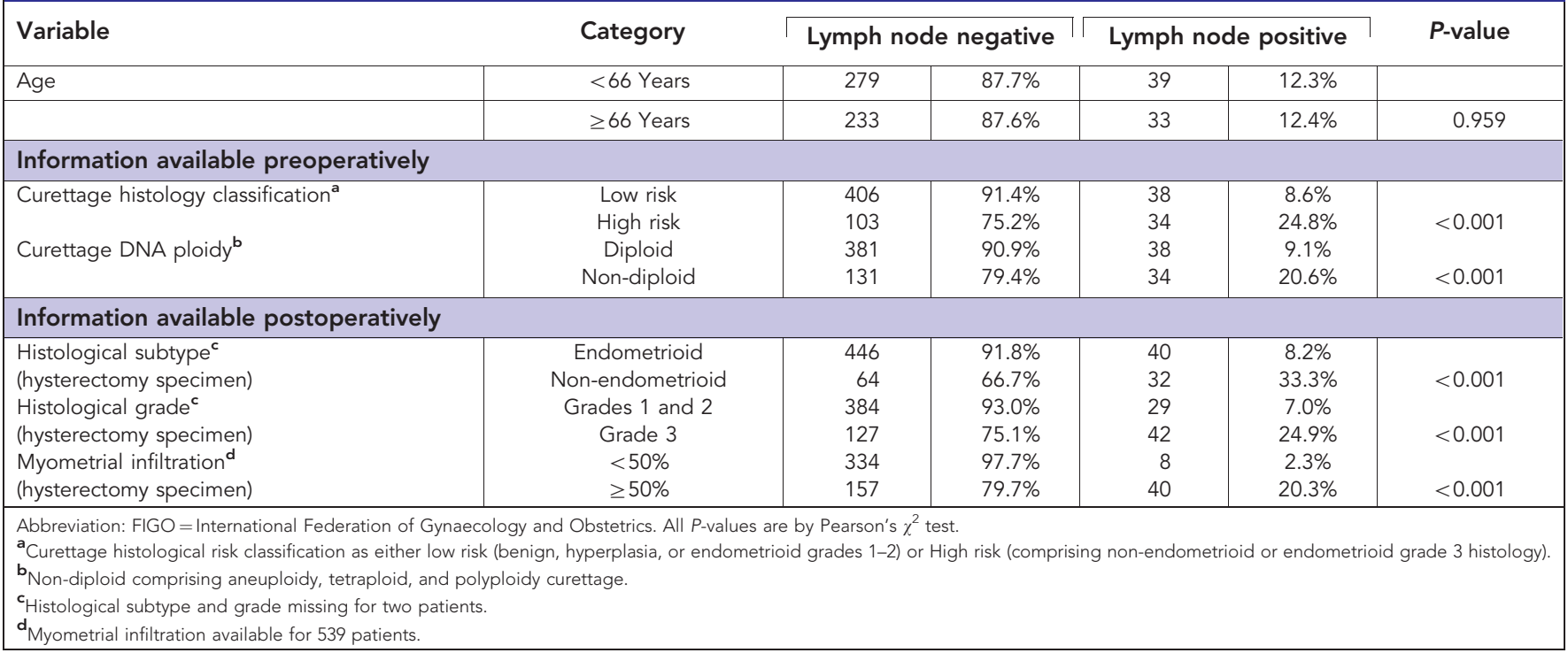

Subgroup analysis was also performed according to lymph node status (Supplementary Table 2). Comparing ploidy status isolated to patients with positive lymph node status $(n=66)$, negative lymph node status $(n=485)$, or unevaluated lymph node status $(n=182)$, a significant reduction in 5-year DSS for non-diploid status was observed among the latter two. The 5-year survival proportions for diploid $v s$ non-diploid curettages were $59.0 \%$ vs $55.2 \%$ for patients with positive lymph node status (Figure $2 \mathrm{~A}$, $P=0.680), 95.5 \%$ vs $88.3 \%$ for patients with negative lymph node status $(P=0.003$, Figure $2 \mathrm{~B})$, and $80.5 \%$ vs $47.2 \%$ for patients with unevaluated lymph node status $(P<0.001$, Figure $2 \mathrm{C})$. Furthermore, considering patients with FIGO stage I/II only and concomitant unevaluated lymph node status only, the prognostic effect of non-diploid status remained. Among lymph node unevaluated patients with localised disease, the survival proportions were significantly lower for patients with non-diploid vs diploid curettage, with 5-year DSS of $93.4 \%$ and $62.0 \%$ respectively (Figure 2D, $P=0.001$ ).

Results from multivariate Cox regression analyses are listed in Supplementary Table 3. Unadjusted for other parameters, nondiploid curettage was associated with a significant increase in risk of disease-specific death among FIGO stage I-III patients (unadjusted $\mathrm{HR}$ of $3.63, P<0.001$ ). When adjusting for preoperatively available curettage risk classification, non-diploid curettage status did not remain significant, but showed a $62 \%$ increased risk of disease-related death (HR 1.62, 95\% CI: 0.90-2.92, $P=0.111$ ). 

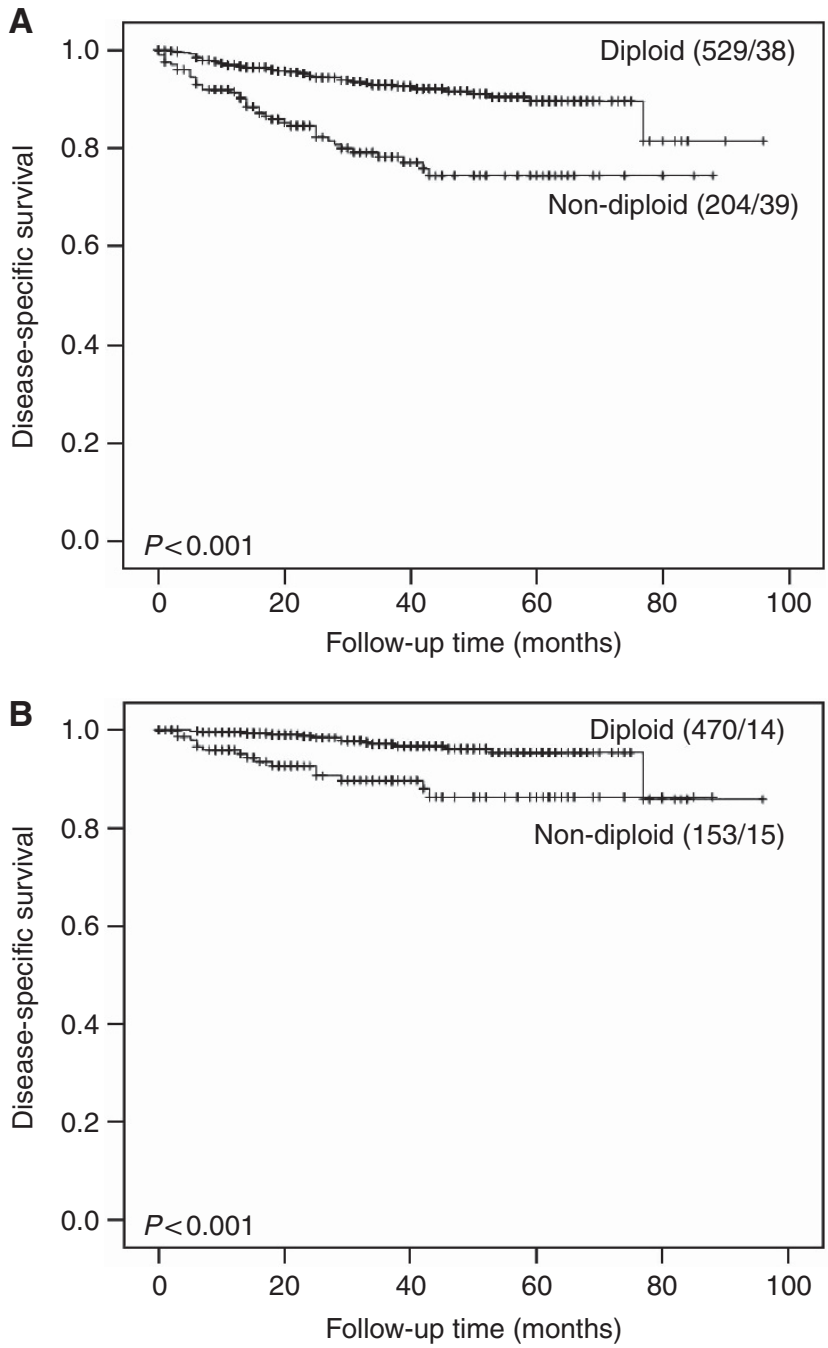

Figure 1. Disease-specific survival (DSS) for endometrial cancer patients related to curettage specimens classified according to DNA ploidy. (A) Disease-specific survival for all patients, grouped according to DNA ploidy $(n=733)$. (B) Disease-specific survival for patients with FIGO stage I/II according to curettage DNA ploidy $(n=623)$. Patients with aneuploid, tetraploid, and polyploid tumours were merged because of similar survival, and classified as non-diploid. The number of cases followed by the number of endometrial carcinoma-related deaths is given in parenthesis. $P$-values are by Kaplan-Meier estimation by the log-rank test.

curettage specimens to be an independent prognostic factor; however, results should be interpreted with care as DNA ploidy was only bivariately corrected for u-PA (urokinase plasminogen activator) or PAI-1 (plasminogen activator inhibitor type 1) (Fredstorp-Lidebring et al, 2001). Furthermore, a study exploring DNA ploidy and lymph node status for 82 endometrial cancer patients found no significant association (Mariani et al, 2005). Our study is thus an important supplement to this inconclusive exploration of the significance of curettage DNA ploidy status.

Noticeably, although the prognostic value of DNA ploidy in curettage specimens perhaps may be disregarded as inferior to hysterectomy samples, the strength of being preoperatively accessible before hysterectomy bears an immediate advantage as a possible supplement to preoperative risk assessment and prediction of lymph node metastasis. In this regard, our perhaps most important findings are that among our patient cohort without distant metastasis at diagnosis, DNA ploidy is an independent predictor of lymph node metastasis, corrected for routinely performed curettage histological risk stratification (OR $1.94, P=0.033)$. For this analysis we excluded cases with FIGO stage IV metastatic disease, as their distant metastasis can be detected during preoperative work-up, and as they have poor prognosis and are subjected to highly individualised treatment strategies also dependent on comorbidity. FIGO stage II and III cases were included to avoid selection bias because of postoperative upstaging in a population with a high proportion of patients subjected to staging lymphadenectomy. Curettage risk stratification showed a stronger trend towards positive lymph node status (OR 2.23, $P=0.011$ ), although it may be considered a more subjective parameter. In this regard, contemplating DNA ploidy and histological risk stratification with other curettage biomarkers such as p53 and hormonal receptor status would be of interest, in particular as the latter methods are more applicable in a routine diagnostic setting. In our subgroup analysis portrayed in Supplementary Table 2, we illustrate that DNA ploidy could be of use in a subset of patients to prognosticate disease behaviour, such as patients with unevaluated lymph node status. Noticeably, DNA ploidy was not significantly associated with DSS for patients with aggressive phenotype such as high FIGO stage and confirmed positive lymph node status. However, for patients with a presumably less aggressive disease such as low FIGO stage or negative lymph node status, patients with non-diploid tumours had significantly shorter DSS. The observation among patients not subjected to lymphadenectomy, where non-diploid curettage status was associated with shorter DSS, supports a particular relevance for DNA ploidy assessment among patients with unevaluated lymph node status. Our findings could be confounded by patient operability; however, excluding patients with high FIGO stage did not alter the significance of our results (Figure 2D). Our data suggest that DNA ploidy evaluated in curettage specimens can represent an important clinical tool in risk stratification compared with surgical lymph node staging with documented short- and long-term side effects (Amant et al, 2005; Salvesen et al, 2012).

As noted, evaluation of DNA ploidy in postoperatively acquired hysterectomy samples may be viewed as superior to curettage specimens. This was exemplified in a comprehensive study by Pradhan et al (2010), including patients with stage I and II endometrioid adenocarcinoma, concluding that analysis of hysterectomy specimen exceeded curettage specimens in predicting biological behaviour. As a possible explanation they postulate that the biology of the deep infiltrating parts of the tumour, of which samples are obtained after hysterectomy, are more important for recurrence development than the superficial part of the tumour, obtained by curettage. As a consequence of intratumoural heterogeneity, DNA ploidy classification may differ between hysterectomy and curettage specimens, as illustrated in a study comparing the two modalities (Pradhan et al, 2010). In an extensive study exploring the genomic landscape of primary renal cell carcinoma, Gerlinger et al (2012) conclude that the intratumoural heterogeneity undetected by single-tumour biopsy samples may present major challenges to personalised medicine and biomarker development. This also directly applies to endometrial cancer, where any curettage sample evidently is restricted to not only represent a distinct area of the endometrium, but also a limited fraction of a tumour with potentially heterogeneous characteristics. This is an aspect that must be taken into consideration when implementing curettage biomarkers into clinical practice, including the reproducibility challenges faced with the standardly applied histological typing and grading (Salvesen et al, 2012). On the other hand, DNA ploidy may in this regard be characterised as a robust method taking intratumoural heterogeneity into account compared with other molecular markers, as a large amount of cell nuclei are measured with a high sensitivity of detecting subgroup aberrations. 

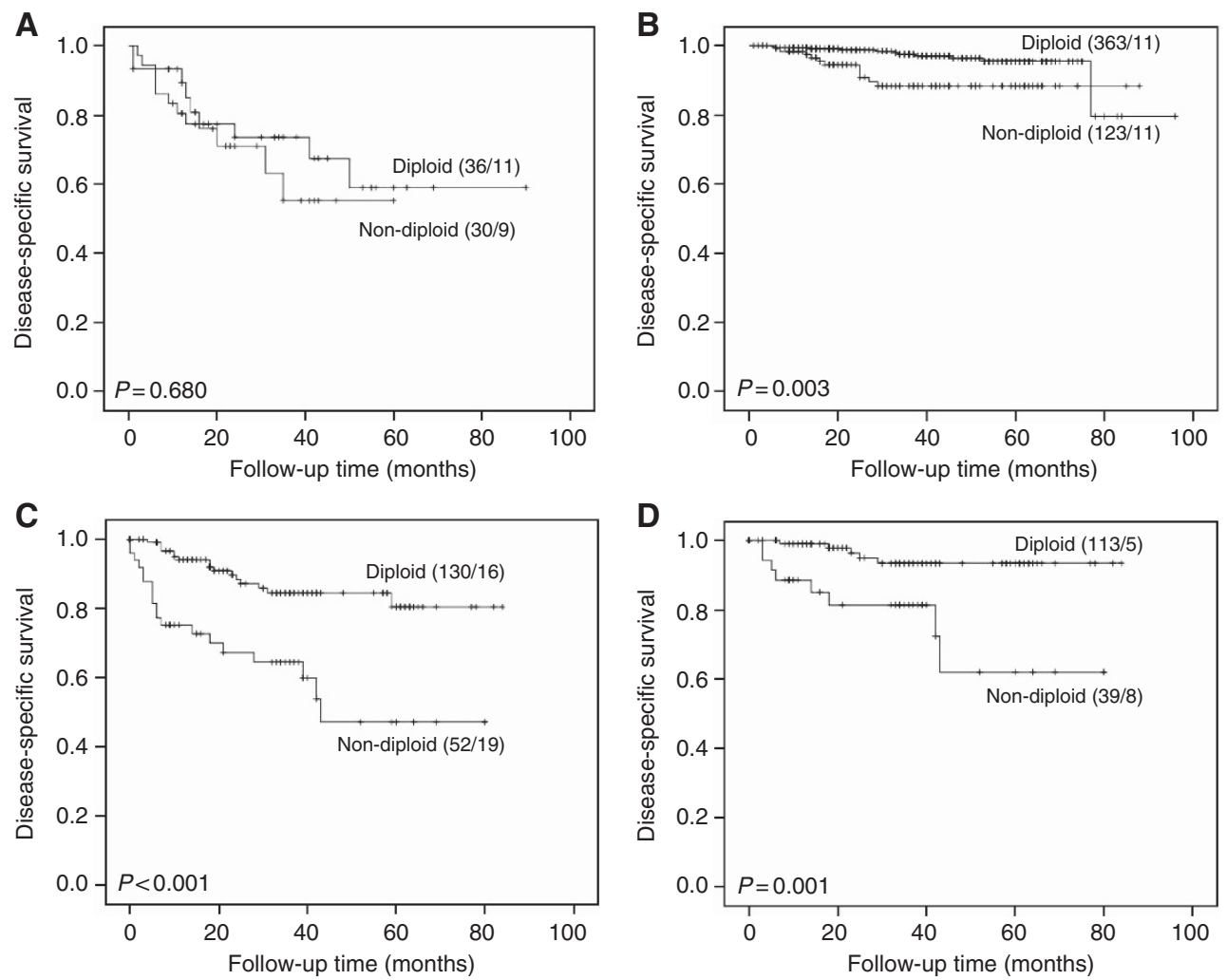

Figure 2. Disease-specific survival (DSS) for endometrial cancer patients in relation to curettage specimen DNA ploidy and lymph node status. (A) Disease-specific survival for patients with positive lymph node status $(n=66)$; (B) DSS for patients with negative lymph node status ( $n=485)$; (C) DSS for patients with unevaluated lymph node status ( $n=182)$; (D) DSS for patients with FIGO stage I/II and unevaluated lymph node status $(n=152)$. Patients with aneuploid, tetraploid, and polyploid tumours were merged because of similar survival, and classified as non-diploid.

The number of cases followed by the number of endometrial carcinoma related deaths is given in parenthesis. All P-values are by Kaplan-Meier estimation by the log-rank test.

Tumour aneuploidy is a common genetic aberration in cancer reflecting a trait of genetic instability. Whether this aneuploidisation is a cause or consequence of malignant transformation is uncertain (Holland and Cleveland, 2009). As opposed to structural chromosome rearrangements such as deletions, amplifications, or translocations, the role of whole-chromosome aneuploidy in cancer has received less attention (Pellman, 2007; Gordon et al, 2012). Aneuploidy has long been hypothesised as a promoter of tumourigenesis (Boveri, 1914). However, recent papers have shed light on the potential activation of oncogenes and inactivation of tumour suppressor genes leading to aneuploidy (Solomon et al, 2011), suggesting aneuploidy as a passenger of tumourigenesis. Aneuploidy is no obligatory trait of cancer, nor is cancer an obligatory consequence of aneuploidy. In concordance with other genetic instability, the pathways involved and the genetic context is of importance in developing the cancerous phenotype. The challenge remains to identify such genetic fingerprints, and translating results to individualise treatment, optimising patient outcome.

Our study is not without limitations. As noted, of the 1046 patients selected for DNA ploidy analysis, only 810 were successfully ploidy classified because of aforementioned reasons. This exclusion of 236 patients could cause a potential selection bias affecting our results. However, testing patients excluded from the study in terms of clinicopathological characteristics displayed in Table 1, no significant difference was observed in comparison with our final study cohort (all $P>0.05$, results not shown). In addition, the proportion of patients unamenable for classification can limit the value of DNA ploidy assessment in routine use, calling for a method with higher rate of successful classification. Our approach of obtaining DNA ploidy material by punching out areas with highest tumour grade may pose a selection bias towards enriching for the more aggressive regions of the tumour in case of heterogeneity. However, this method allows small samples to be analysed, applicable to small endometrial biopsies. Our study cohort portrays a proportion of patients subjected to lymphadenectomy of $74.4 \%$, illustrating a challenge complying with a staging procedure including lymphadenectomy in an elderly and often comorbid patient population. The extent of lymph node dissection was decided by the responsible surgeon, balancing patient comorbidity and risk involved in extended surgery. As expected, patients subjected to lymphadenectomy were significantly younger, and no other systematic biases were observed (Supplementary Table 1). This suggests a selection bias of patients subjected to lymph node sampling based on age, but not the other investigated biomarkers.

In conclusion, our study demonstrates that non-diploid curettage samples are associated with aggressive phenotype, lymph node metastasis, and poor DSS. With the significant association between DNA ploidy and lymph node status, DNA ploidy can be of interest preoperatively to identify high-risk patients for tailored surgical treatment. Furthermore, subgroups of patients with presumably less aggressive traits, such as low FIGO stage and negative lymph node status from staging lymphadenectomy, have significantly shorter DSS in the case of non-diploid curettage sample. This prognostic effect was also observed among patients not subjected to staging lymphadenectomy. Our results indicate DNA ploidy as a possible supplement to existing parameters used to tailor surgical treatment such as curettage histological risk stratification. However, additional studies, and in particular a prospective randomised clinical trial, would be important to evaluate the effect of implementing DNA ploidy into routine clinical practice. 


\section{ACKNOWLEDGEMENTS}

We thank Britt Edvardsen for technical assistance. We also thank participants responsible for recruiting patients at the other MoMaTEC (Molecular Markers in Treatment of Endometrial Cancer) centres. This work was supported by the Helse Vest Research Fund; the Norwegian Research Council; the Norwegian Cancer Society (The Harald Andersen's legacy); and by the Research Council of Norway through its Centres of Excellence funding scheme, project number 179571. Frederic Amant is senior researcher for the Research Fund-Flanders (FWO). The funding sponsors had no role in the study design, collection, analysis or interpretation of data, nor in writing the report.

\section{CONFLICT OF INTEREST}

The authors declare no conflict of interest.

\section{REFERENCES}

American Cancer Society (2014) Cancer Facts \& Figures 2014.

Abeler VM, Kjorstad KE (1991) Endometrial adenocarcinoma in Norway. A study of a total population. Cancer 67(12): 3093-3103.

Amant F, Moerman P, Neven P, Timmerman D, Van Limbergen E, Vergote I (2005) Endometrial cancer. Lancet 366(9484): 491-505.

Boveri T (1914) The Origin of Malignant Tumors. The Williams and Wilkins Company: Baltimore.

Cancer Registry of Norway (2012) Cancer in Norway. Cancer incidence, mortality, survival and prevalence in Norway.

Creutzberg CL, van Putten WL, Koper PC, Lybeert ML, Jobsen JJ, Wárlám-Rodenhuis CC, De Winter KA, Lutgens LC, van den Bergh AC, van de Steen-Banasik E (2000) Surgery and postoperative radiotherapy versus surgery alone for patients with stage-1 endometrial carcinoma: multicentre randomised trial. Lancet 355(9213): 1404-1411.

Fredstorp-Lidebring M, Bendahl P-O, Brünner N, Casslen B, Högberg T, Långström-Einarsson E, Willén R, Fernö M (2001) Urokinase plasminogen activator and its inhibitor, PAI-1, in association with progression-free survival in early stage endometrial cancer. Eur J Cancer 37(18): 2339-2348.

Gerlinger M, Rowan AJ, Horswell S, Larkin J, Endesfelder D, Gronroos E, Martinez P, Matthews N, Stewart A, Tarpey P (2012) Intratumor heterogeneity and branched evolution revealed by multiregion sequencing. $N$ Engl J Med 366(10): 883-892.

Gordon DJ, Resio B, Pellman D (2012) Causes and consequences of aneuploidy in cancer. Nat Rev Genet 13(3): 189-203.

Holland AJ, Cleveland DW (2009) Boveri revisited: chromosomal instability, aneuploidy and tumorigenesis. Nat Rev Mol Cell Biol 10(7): 478-487.

Jemal A, Siegel R, Xu J, Ward E (2010) Cancer statistics, 2010. CA Cancer J Clin 60(5): 277-300.

Larson DM, Berg R, Shaw G, Krawisz BR (1999) Prognostic significance of DNA ploidy in endometrial cancer. Gynecol Oncol 74(3): 356-360.

Lundgren C, Auer G, Frankendal B, Nilsson B, Nordström B (2004) Prognostic factors in surgical stage I endometrial carcinoma. Acta Oncol 43(1): 49-56.

Mariani A, Sebo TJ, Katzmann JA, Keeney GL, Roche PC, Lesnick TG, Podratz KC (2000) Pretreatment assessment of prognostic indicators in endometrial cancer. Am J Obstet Gynecol 182(6): 1535-1544.

Mariani A, Sebo TJ, Katzmann JA, Roche PC, Keeney GL, Lesnick TG, Podratz KC (2005) Endometrial cancer: can nodal status be predicted with curettage? Gynecol Oncol 96(3): 594-600.

Mauland KK, Wik E, Salvesen HB (2014) Clinical value of DNA content assessment in endometrial cancer. Cytometry B Clin Cytom 86(3): 154-163.
Merkel DE, McGuire WL (1990) Ploidy, proliferative activity and prognosis. DNA flow cytometry of solid tumors. Cancer 65(5): 1194-1205.

Pellman D (2007) Cell biology: aneuploidy and cancer. Nature 446(7131): 38-39.

Pfisterer J, Kommoss F, Sauerbrei W, Rendl I, Kiechle M, Kleine W, Pfleiderer A (1995) Prognostic value of DNA ploidy and S-phase fraction in stage I endometrial carcinoma. Gynecol Oncol 58(2): 149-156.

Pradhan M, Abeler V, Danielsen H, Sandstad B, Tropé C, Kristensen G, Risberg B (2012) Prognostic importance of DNA ploidy and DNA index in stage I and II endometrioid adenocarcinoma of the endometrium. Ann Oncol 23(5): 1178-1184.

Pradhan M, Abeler VM, Danielsen HE, Tropé CG, Risberg BÅ (2006) Image cytometry DNA ploidy correlates with histological subtypes in endometrial carcinomas. Mod Pathol 19(9): 1227-1235.

Pradhan M, Abeler VM, Davidson B, Kildal W, Nybøen Å, Tropé CG, Risberg B, Danielsen HE (2010) DNA ploidy heterogeneity in endometrial carcinoma: comparison between curettage and hysterectomy specimens. Int J Gynecol Pathol 29(6): 572-578.

Prat J (2004) Prognostic parameters of endometrial carcinoma. Hum Pathol 35(6): 649-662.

Salvesen HB, Akslen LA (2002) Molecular pathogenesis and prognostic factors in endometrial carcinoma. APMIS 110(10): 673-689.

Salvesen HB, Haldorsen IS, Trovik J (2012) Markers for individualised therapy in endometrial carcinoma. Lancet Oncol 13(8): e353-e361.

Siegel R, Ma J, Zou Z, Jemal A (2014) Cancer statistics, 2014. CA Cancer J Clin 64(1): 9-29.

Solomon DA, Kim T, Diaz-Martinez LA, Fair J, Elkahloun AG, Harris BT, Toretsky JA, Rosenberg SA, Shukla N, Ladanyi M (2011) Mutational inactivation of STAG2 causes aneuploidy in human cancer. Science 333(6045): 1039-1043.

Sorosky JI (2012) Endometrial cancer. Obstet Gynecol 120(2, Part 1): 383-397. Steinbakk A, Malpica A, Slewa A, Skaland I, Gudlaugsson E, Janssen EA, Løvslett K, Fiane B, Kruse AJ, Feng W (2011) Biomarkers and microsatellite instability analysis of curettings can predict the behavior of FIGO stage I endometrial endometrioid adenocarcinoma. Mod Pathol 24(9): 1262-1271.

Susini T, Amunni G, Molino C, Carriero C, Rapi S, Branconi F, Marchionni M, Taddei G, Scarselli G (2007) Ten-year results of a prospective study on the prognostic role of ploidy in endometrial carcinoma. Cancer 109(5): $882-890$.

Terada K (2012) DNA ploidy in endometrial cancer: unfinished business? Ann Oncol 23(5): 1083-1084.

Trovik J, Mauland KK, Werner HM, Wik E, Helland H, Salvesen HB (2012) Improved survival related to changes in endometrial cancer treatment, a 30-year population based perspective. Gynecol Oncol 125(2): 381-387.

Trovik J, Wik E, Stefansson IM, Marcickiewicz J, Tingulstad S, Staff AC, Njolstad TS, Vandenput I, Amant F, Akslen LA (2011) Stathmin overexpression identifies high-risk patients and lymph node metastasis in endometrial cancer. Clin Cancer Res 17(10): 3368-3377.

Trovik J, Wik E, Werner HM, Krakstad C, Helland H, Vandenput I, Njolstad TS, Stefansson IM, Marcickiewicz J, Tingulstad S, Staff AC, Amant F, Akslen LA, Salvesen HB (2013) Hormone receptor loss in endometrial carcinoma curettage predicts lymph node metastasis and poor outcome in prospective multicentre trial. Eur J Cancer 49: 3431-3441.

Wik E, Trovik J, Iversen OE, Engelsen IB, Stefansson IM, Vestrheim LC, Haugland HK, Akslen LA, Salvesen HB (2009) Deoxyribonucleic acid ploidy in endometrial carcinoma: a reproducible and valid prognostic marker in a routine diagnostic setting. Am J Obstet Gynecol 201: 603. e1-603. e7.

Zaino RJ, Davis AT, Ohlsson-Wilhelm BM, Brunetto VL (1998) DNA content is an independent prognostic indicator in endometrial adenocarcinoma. a Gynecologic Oncology Group study. Int J Gynecol Pathol 17(4): 312-319.

This work is published under the standard license to publish agreement. After 12 months the work will become freely available and the license terms will switch to a Creative Commons AttributionNonCommercial-Share Alike 4.0 Unported License.

Supplementary Information accompanies this paper on British Journal of Cancer website (http://www.nature.com/bjc) 


\section{APPENDIX}

This study was conducted within ENITEC (European Network for Individualized Treatment of Endometrial Cancer) with the following participating centres in the MoMaTEC (Molecular

Markers in Treatment of Endometrial Cancer) trial: Norwegian centres: Haukeland University Hospital, Bergen; St. Olav's Hospital, Trondheim; Oslo University Hospital, Ullevål, Oslo; Akershus University Hospital, Oslo; Haugesund Hospital; Hospital of Vestfold; Førde Hospital; Ålesund Hospital; University Hospital Gasthuisberg Leuven, Belgium; and Sahlgrenska Academy, Sweden. 